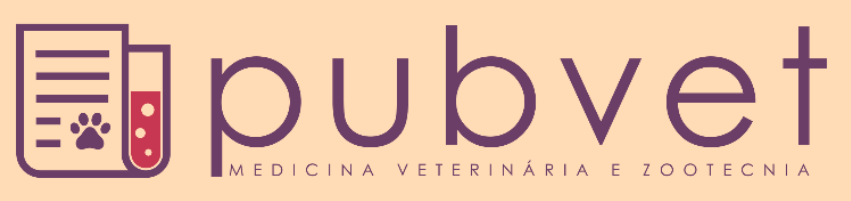

https://doi.org/10.31533/pubvet.v13n4a312.1-12

\title{
Estudo retrospectivo de neoplasmas em animais de companhia atendidos no hospital de clínicas veterinárias da universidade federal de Pelotas durante 2013 a 2017
}

\author{
Daniele Vitor Barboza ${ }^{*} \bullet$, Caroline Xavier Grala ${ }^{2} \bullet$, Edgar Cleiton Da Silva ${ }^{2} \bullet$, Jéssica Paola \\ Salame $^{1 \oplus}$, Andreza Bernardi ${ }^{2}$, Carina Burket Da Silva ${ }^{3}$, Thomas Normanton Guim ${ }^{4} \odot$ \\ 'Residente em Clínica Médica de Animais de Companhia da Universidade Federal de Pelotas \\ ${ }^{2}$ Graduanda em Medicina Veterinária da Universidade Federal de Pelotas \\ ${ }^{3}$ Residente em Diagnóstico por Imagem em Medicina Veterinária da Universidade Federal de Pelotas \\ ${ }^{4}$ Médico Veterinário Doutor Técnico Administrativo da Universidade Federal de Pelotas \\ *Autor para correspondência, E-mail: danielevitorbarboza@gmail.com
}

\begin{abstract}
Resumo. O câncer é uma das principais causas de morte em cães, estudos estimam que $45 \%$ dos animais com mais de 10 anos morrem desta enfermidade. Existem fatores genéticos e ambientais que podem estar envolvidos na ocorrência da doença. Sistemas de coleta de dados são raros em Medicina Veterinária, porém de grande valia para o entendimento dos fatores predisponentes e frequência dos diferentes tumores. Por desenvolverem a doença naturalmente e compartilharem do mesmo ambiente e estilo de vida dos seus tutores, os animais podem ser bons modelos de estudo da ocorrência natural de diversas neoplasias que também acometem humanos. O presente trabalho realizou um levantamento da frequência de 764 animais com queixa de tumores e que tiveram diagnóstico obtido através de exame citológico ou histopatológico no período de 2013 a 2017 no HCV-UFPel. Os tumores foram divididos em 13 grupos de acordo com os sítios anatômicos, em uma classificação baseada no ICD-O-3. O presente trabalho tem por objetivo fornecer dados para futuros epidemiológicos em oncologia veterinária e comparada. Os grupos mais frequentes foram o de tumores da pele e subcutâneo $(40,84 \%)$, seguido pelos tumores das glândulas mamárias $(27,88 \%)$, tumores dos órgãos sexuais e sistema reprodutor $(9,95 \%)$ e tumores do sangue e sistema hemolinfático $(6,54 \%)$.
\end{abstract}

Palavras-chave: Câncer, oncologia, cães, gatos, frequência, ocorrência natural

\section{Retrospective study of neoplasms in company animals attended at the veterinary clinics hospital of the federal university of Pelotas during 2013 to 2017}

Cancer is one of the leading causes of death in dogs, studies estimate that $45 \%$ of animals over 10 years die of this disease. There are genetic and environmental factors that may be involved in the occurrence of the disease. Data collection systems are rare in Veterinary Medicine, but of great value for the understanding of the predisposing factors and frequency of the different tumors. By developing the disease naturally and sharing the same environment and lifestyle of their tutors, animals can be good models of the natural occurrence of various neoplasms that also affect humans. The present work carried out a survey of the frequency of 764 animals complaining of tumors and that had a diagnosis obtained by cytological or histopathological examination in the period from 2013 to 2017 in the HCV-UFPel. Tumors were divided into 13 groups according to the anatomical sites, in a classification based on ICD-O-3. The present work aims to provide data for 
epidemiological future in veterinary and comparative oncology. The most frequent groups were skin and subcutaneous tumors $(40.84 \%)$, followed by tumors of the mammary glands $(27.88 \%)$, tumors of the sexual organs and the reproductive system $(9.95 \%)$ and tumors of the blood and hemolytic system $(6.54 \%)$.

Keywords: Cancer, animals, frequency, natural occurrence

\section{Estudio retrospectivo de neoplasmas en perros y gatos además en el hospital de clínicas veterinarias de la universidad federal de Pelotas durante 2013 a 2017}

Resumen. El cáncer es una de las principales causas de muerte en los perros, los estudios
estiman que el $45 \%$ de los animales mayores de 10 años mueren de esta enfermedad.
Existen factores genéticos y ambientales que pueden estar involucrados en la aparición de
la enfermedad. Los sistemas de recolección de datos son raros en Medicina Veterinaria,
pero son de gran valor para la comprensión de los factores predisponentes y la frecuencia
de los diferentes tumores. Al desarrollar la enfermedad de forma natural y compartir el
mismo entorno y estilo de vida de sus tutores, los animales pueden ser buenos modelos de
la aparición natural de diversas neoplasias que también afectan a los humanos. El presente
trabajo realizó un estudio de la frecuencia de 764 animales que se quejaban de tumores y
que tenía un diagnóstico obtenido por examen citológico o histopatológico en el período
de 2013 a 2017 en el HCV-UFPel. Los tumores se dividieron en 13 grupos según los sitios
anatómicos, en una clasificación basada en ICD-O-3. El presente trabajo tiene como
objetivo proporcionar datos para el futuro epidemiológico en oncología veterinaria y
comparativa. Los grupos más frecuentes fueron tumores cutáneos y subcutáneos (40,84\%),
seguidos de tumores de las glándulas mamarias (27,88\%), tumores de los órganos sexuales
y del sistema reproductor (9,95\%) y tumores de la sangre y del sistema hemolítico $(6,54 \%)$.

Palabras clave: Cáncer, oncología, perros, gatos, frecuencia, ocurrencia natural

\section{Introdução}

A população de animais de estimação tem aumentado cada vez mais em todo o mundo. Segundo o último censo do Instituto Brasileiro de Geografia e Estatística de 2013, o Brasil tem a quarta maior população de animais de estimação do mundo, cerca de 132 milhões de animais. Tal aumento na população vem acompanhado de um estreitamento da relação do homem com esses animais, e consequentemente dos cuidados com os mesmos, culminando com o aumento da longevidade destes pacientes e aumento do aparecimento de doenças crônicas como o câncer (Demetriou \& Foale, 2011; Withrow et al., 2013). Atualmente o câncer é uma das principais causas de morte de pequenos animais. Estudos demonstram que 45\% dos cães com 10 anos ou mais morrem de câncer (Withrow et al., 2013). A prevalência desta doença é cada vez maior, e a cada dia surgem novas opções terapêuticas e formas de manejo para melhorar a qualidade de vida destes pacientes (Daleck et al., 2016). Sabe-se que a patogenia das neoplasias está relacionada a fatores genéticos e ambientais. Todavia, acredita-se que os fatores ambientais sejam os mais significativos. Diferenças na incidência e mortalidade de determinados tipos de câncer em humanos demonstram o importante papel dos fatores ambientais. Os estudos epidemiológicos auxiliam na determinação dos fatores de risco e causas do câncer (Ferreira Neto et al., 1977; Núñez \& Bouda, 2007).

A coleta de dados de pessoas com câncer iniciou em 1900 e foi evoluindo com o decorrer do tempo e percepção da importância dessas informações. Em 1950, os primeiros dados foram tabulados e a prevalência de neoplasias foi publicada pela International Agency for Research on Cancer (IARC). Já em animais, o sistema de coleta de informações de forma padronizada ainda não é realizado. Todavia, é muito difícil estimar a prevalência e a incidência de neoplasmas em animais, porque não há um controle efetivo da população destes. São raros os centros de notificação da ocorrência dessa doença em cães e gatos (Grüntzig et al., 2015; Merlo et al., 2008). Estudar a prevalência e a incidência das neoplasias em animais de companhia não é importante apenas do ponto de vista médico-veterinário, mas pode ter 
também grande relevância para a medicina humana. O estudo da oncologia em animais experimentais é bastante limitado. Muitas vezes o comportamento natural de determinados neoplasmas é diferente se comparado àqueles induzidos em modelos experimentais. Os cães compartilham o mesmo ambiente e são expostos a situações semelhantes às de seus tutores. Além de possuírem grande variabilidade genética, muitas neoplasias acometem ambas as espécies. Dessa maneira, o cão é um modelo importante de ocorrência natural e estudo do câncer (Hayes Junior, 1978; Rowell et al., 2011).

\section{Material e métodos}

Foi realizado um estudo retrospectivo dos prontuários clínicos de pacientes oncológicos atendidos no HCV-UFPel com diagnóstico confirmado por meio de exame citológico ou histopatológico durante o período de janeiro de 2013 a dezembro de 2017. Alterações benignas e malignas foram incluídas no estudo.

Foram avaliados a espécie, a idade, a raça, o sexo, a localização e o tipo histológico das neoformações dos animais. Os neoplasmas foram divididos em grupos de acordo com a localização. A classificação foi adaptada da International Classification of Diseases for Oncology, terceira revisão (ICD-O-3). O ICD constitui um sistema de classificação de tumores conhecido internacionalmente, que busca dividir os mesmos de acordo com a sua topografia e morfologia. O ICD-O-3 classifica de acordo com sítios anatômicos genéricos (Gjerstorff, 2011; Grüntzig et al., 2015; Kavuluru et al., 2013).

\section{Resultados e discussão}

No período de janeiro de 2013 a dezembro de 2017 foram atendidos 764 animais com queixa de neoplasmas, que tiveram diagnóstico realizado por meio de exame citológico ou histopatológico. Deste total, $683(89,4 \%)$ animais eram caninos e $81(10,6 \%)$ eram felinos. Na Figura 1 observamos o sexo dos animais atendidos de acordo com a espécie.

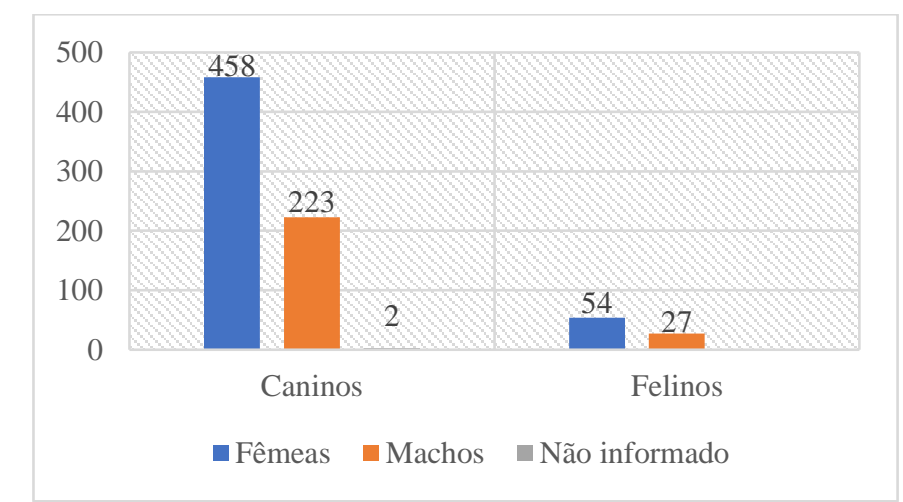

Figura 1. Sexo dos caninos e felinos atendidos no HCV-UFPel com diagnóstico de doença oncológica, no período de 2013 a 2017.

Conforme observado na Figura 1, as fêmeas foram mais frequentes em ambas as espécies. Dois caninos $(0,29 \%)$ não tiveram o sexo identificado na ficha clínica. A maior frequência de fêmeas corrobora com os achados descritos por De Nardi et al. (2002). A média de idade dos cães foi de 8,5 \pm 4,7 anos e dos gatos foi de 7,0 $\pm 4,4$ anos. $O$ cão mais jovem atendido com neoplasma tinha sete meses e o mais idoso 21 anos. Já o gato o mais jovem tinha sete meses e o mais idoso 17 anos. A maior parte dos animais acometidos por câncer é idoso, assim como observado por Withrow et al. (2013). Este fato pode ser explicado devido alterações que podem ocorrer nas vias de controle de crescimento e replicação celular com o passar do tempo levando ao surgimento de neoplasmas (Campisi, 2003; Withrow et al., 2013). Animais idosos podem ter um acúmulo de células senescentes em certos tecidos predispondo a ocorrência de neoplasmas (Campisi, 2003). Os cães sem raça definida foram os mais acometidos por neoplasmas neste estudo, seguido dos cães da raça Poodle e Labrador. Entre os felinos, os sem raça definida também tiveram maior frequência de neoplasmas. Estes resultados provavelmente estão associados ao fato de que no HCV-UFPel a maior parte dos animais atendidos é sem raça definida. Na tabela 1 e 2 estão demonstradas as raças dos animais do estudo. 
Tabela 1. Raças de caninos diagnosticados com doença oncológica no HCV-UFPel, no período de 2013 a 2017

\begin{tabular}{|c|c|c|}
\hline Raças & Número, $\mathrm{n}$ & Frequência, \% \\
\hline$\overline{\text { Sem raça definida }}$ & 358 & 52,41 \\
\hline Poodle & 42 & 6,15 \\
\hline Labrador & 40 & 5,85 \\
\hline Pitbull & 33 & 4,83 \\
\hline Daschund & 30 & 4,41 \\
\hline Rottweiler & 21 & 3,07 \\
\hline Boxer & 21 & 3,07 \\
\hline Cocker spaniel & 16 & 2,34 \\
\hline Yorkshire & 14 & 2,04 \\
\hline Pastor alemão & 13 & 1,90 \\
\hline Pinscher & 12 & 1,75 \\
\hline Pointer & 6 & 0,88 \\
\hline Golden Retriver & 6 & 0,88 \\
\hline Dálmata & 6 & 0,88 \\
\hline Buldogue inglês & 6 & 0,88 \\
\hline Beagle & 5 & 0,73 \\
\hline Doberman & 4 & 0,58 \\
\hline Collie & 4 & 0,58 \\
\hline Shi-tzu & 3 & 0,44 \\
\hline Ovelheiro gaúcho & 3 & 0,44 \\
\hline Galgo & 3 & 0,44 \\
\hline Fox terrier & 3 & 0,44 \\
\hline Buldogue campeiro & 3 & 0,44 \\
\hline Schnauzer & 2 & 0,29 \\
\hline Não informado & 2 & 0,29 \\
\hline Maltês & 2 & 0,29 \\
\hline Dogo argentino & 2 & 0,29 \\
\hline Chow- chow & 2 & 0,29 \\
\hline Bull terrier & 2 & 0,29 \\
\hline Border collie & 2 & 0,29 \\
\hline Akita & 2 & 0,29 \\
\hline Whippet & 1 & 0,15 \\
\hline Setter irlandês & 1 & 0,15 \\
\hline São Bernardo & 1 & 0,15 \\
\hline Rodhesian ridgeback & 1 & 0,15 \\
\hline Pug & 1 & 0,15 \\
\hline Pastor suíço & 1 & 0,15 \\
\hline Pastor belga & 1 & 0,15 \\
\hline Lhasa apso & 1 & 0,15 \\
\hline Fila brasileiro & 1 & 0,15 \\
\hline Dogue alemão & 1 & 0,15 \\
\hline Cimarron uruguaio & 1 & 0,15 \\
\hline Cane corso & 1 & 0,15 \\
\hline Buldogue francês & 1 & 0,15 \\
\hline Bichon frise & 1 & 0,15 \\
\hline Australian cattle dog & 1 & 0,15 \\
\hline Total & 683 & 100 \\
\hline
\end{tabular}


Tabela 2. Raças de felinos diagnosticados com doença oncológica no HCV-UFPel no período de 2013 a 2017

\begin{tabular}{lcc}
\hline Raças & Número (n) & Frequência (\%) \\
\hline Sem raça definida & 75 & 92,59 \\
Siamês & 4 & 4,95 \\
Himalaia & 1 & 1,23 \\
Ragdoll & 1 & 1,23 \\
\hline Total & 81 & 100 \\
\hline
\end{tabular}

O grupo de neoplasmas que apresentou a maior frequência de diagnósticos foi o grupo de tumores da pele e subcutâneo, seguido pelos tumores das glândulas mamárias, tumores dos órgãos sexuais e sistema reprodutor e tumores do sangue e sistema hemolinfático. Os dados encontrados são bastante interessantes se comparados à última estimativa para o biênio 2018 e 2019 do Instituto Nacional do Câncer José Alencar Gomes da Silva (INCA) em humanos. Estimou-se que no Brasil as neoplasias mais frequentes serão as neoplasias de pele não melanomas, seguido pelos tumores de mama em mulheres. Já em homens, o panorama do segundo sítio neoplásico mais frequente é diferente, sendo os tumores de próstata os mais frequentes depois dos tumores cutâneos não melanomas. Na tabela 3, verificam-se os grupos de diagnósticos oncológicos e a frequência das afecções nos diferentes grupos.

Tabela 3. Número e frequência dos tumores divididos de acordo com a ICD-O-3 adaptada em caninos e felinos, diagnosticados no HCV-UFPel no período de 2013 a 2017-Continua

\begin{tabular}{lccc}
\hline Grupo & Caninos & Felinos & Total \\
$\mathrm{n}(\%)$
\end{tabular}

O fato das neoformações de pele e subcutâneo representar a maior parte dos diagnósticos $(40,8 \%)$, seguida pelos tumores de glândula mamária $(27,9 \%)$ corrobora com os achados de De Nardi et al. (2002), Withrow et al. (2013) e Grüntzig et al. (2015). A neoplasia cutânea que apresentou a maior frequência foi o mastocitoma. Na tabela 4 observam-se os neoplasmas cutâneos diagnosticados e as respectivas frequências em caninos e felinos.

Os mastocitomas $(23,4 \%)$ foram os tumores mais frequentes seguidos pelos carcinomas de células escamosas (14,4\%). Tais dados corroboram com os achados por Souza et al. (2006) e Lima et al. (2018) que realizaram estudos retrospectivos de neoplasias cutâneas cujos resultados foram semelhantes. A etiologia da maior parte dos tumores cutâneos é desconhecida, incluindo o mastocitoma. Mas, a exposição crônica aos raios ultravioleta do tipo beta está relacionada com o desenvolvimento de carcinoma de células escamosas (Daleck et al., 2016). Em humanos, os tumores de pele são divididos em não melanomas e melanomas. Os tumores de pele não melanomas têm como principal fator predisponente a exposição solar crônica, sendo os carcinomas basocelulares e espinocelulares os mais frequentes nesta espécie (Borsato \& Almeida, 2009). Nota-se a importância da exposição solar na etiopatogenia de alguns tumores cutâneos em ambas as espécies. 
Tabela 4. Tumores da pele e tecido subcutâneo diagnosticados em caninos e felinos no HCV- UFPel, no período de 2013 a 2017

\begin{tabular}{|c|c|c|c|}
\hline Diagnóstico & $\begin{array}{c}\text { Caninos } \\
\mathrm{n}\end{array}$ & $\begin{array}{c}\text { Felinos } \\
\mathrm{n}\end{array}$ & $\begin{array}{l}\text { Total } \\
\mathrm{n}(\%)\end{array}$ \\
\hline Mastocitoma & 73 & - & $73(23,4)$ \\
\hline Carcinoma de células escamosas & 23 & 22 & $45(14,42)$ \\
\hline Lipoma & 30 & - & $30(9,61)$ \\
\hline Fibrossarcoma & 16 & 8 & $24(7,7)$ \\
\hline Hemangiossarcoma & 16 & 1 & $17(5,45)$ \\
\hline Melanoma & 14 & - & $14(4,5)$ \\
\hline Adenoma sebáceo & 9 & - & $9(2,9)$ \\
\hline Hemangioma & 9 & - & $9(2,9)$ \\
\hline Carcinoma ductal apócrino & 7 & - & $7(2,24)$ \\
\hline Hiperplasia sebácea & 6 & 1 & $7(2,24)$ \\
\hline Papiloma & 7 & - & $7(2,24)$ \\
\hline Carcinoma de glândulas ceruminosas & 4 & 2 & $6(1,92)$ \\
\hline Carcinoma hepatoide & 6 & - & $6(1,92)$ \\
\hline Epitelioma sebáceo & 6 & - & $6(1,92)$ \\
\hline Lipossarcoma & 5 & - & $5(1,6)$ \\
\hline Adenoma hepatoide & 4 & - & $4(1,3)$ \\
\hline Sarcoma histiocítico & 4 & - & $4(1,3)$ \\
\hline Adenoma apócrino & 1 & 2 & $3(0,96)$ \\
\hline Carcinoma in situ & 2 & 1 & $3(0,96)$ \\
\hline Histiocitoma & 2 & 1 & $3(0,96)$ \\
\hline Sarcoma de tecido mole & 2 & 1 & $3(0,96)$ \\
\hline Carcinoma de saco anal & 2 & - & $2(0,64)$ \\
\hline Fibroma & 2 & - & $2(0,64)$ \\
\hline Leiomiossarcoma & 2 & - & $2(0,64)$ \\
\hline Osteossarcoma extraesquelético & 2 & - & $2(0,64)$ \\
\hline Sarcoma cutâneo & 2 & - & $2(0,64)$ \\
\hline Tricoblastoma & 2 & - & $2(0,64)$ \\
\hline Adenocarcinoma peniano & 1 & - & $1(0,32)$ \\
\hline Adenoma sebáceo & 1 & - & $1(0,32)$ \\
\hline Carcinoma basocelular & 1 & - & $1(0,32)$ \\
\hline Carcinoma basoescamoso & 0 & 1 & $1(0,32)$ \\
\hline Carcinoma indiferenciado & 1 & - & $1(0,32)$ \\
\hline Carcinoma sebáceo & 1 & - & $1(0,32)$ \\
\hline Epitelioma hepatoide & 1 & - & $1(0,32)$ \\
\hline Hamartoma colagenoso & 1 & - & $1(0,32)$ \\
\hline Hamartoma vascular & 1 & - & $1(0,32)$ \\
\hline Melanocitoma & 1 & - & $1(0,32)$ \\
\hline Piloleiomioma & 1 & - & $1(0,32)$ \\
\hline Tumor de células basais & 1 & - & $1(0,32)$ \\
\hline Tumor de células redondas & 0 & 1 & $1(0,32)$ \\
\hline Tumor venéreo transmissível & 1 & - & $1(0,32)$ \\
\hline Total & 271 & 41 & $312 / 100$ \\
\hline
\end{tabular}

Depois dos tumores cutâneos, os tumores das glândulas mamárias foram os mais frequentes, correspondendo a $27,88 \%$ da casuística estudada. A tabela 5 mostra todos os tumores mamários diagnosticados no período do estudo, bem como a frequência e a espécie acometida.

Até $60 \%$ das cadelas podem ter diferentes tipos histológicos de tumores na mesma mama ou em mamas diferentes (Benjamin et al., 1999). Devido à alta frequência de animais com vários tipos 
histológicos de tumores mamários optou-se pela contabilização em um grupo de animais com tumores mamários múltiplos e não os separar. Desta maneira, foi constatada a frequência alta de animais com mais de um tipo neoplásico mamário.

Tabela 5. Tumores de glândulas mamárias diagnosticados em caninos e felinos no HCV- UFPel no período de 2013 a 2017

\begin{tabular}{lccc}
\hline Diagnóstico & Caninos, $\mathrm{n}$ & Felinos, $\mathrm{n}$ & Total, $\mathrm{n}(\%)$ \\
\hline Tumores mamários múltiplos* & 93 & 3 & $96(45,07)$ \\
Carcinoma** & 20 & 4 & $24(11,27)$ \\
Carcinoma em tumor misto & 22 & 1 & $23(10,8)$ \\
Carcinoma tubular & 11 & 4 & $15(7,04)$ \\
Carcinossarcoma & 14 & - & $14(6,57)$ \\
Adenoepitelioma maligno & 9 & - & $9(4,22)$ \\
Carcinoma complexo & 8 & - & $8(3,76)$ \\
Carcinoma sólido & 5 & 1 & $6(2,82)$ \\
Carcinoma tubulopapilar & 4 & - & $4(1,88)$ \\
Carcinoma papilar & 2 & - & $2(0,94)$ \\
Ectasia ductal & 2 & - & $2(0,94)$ \\
Hiperplasia mamária & 2 & - & $2(0,94)$ \\
Carcinoma anaplásico & 1 & $1(0,47)$ \\
Carcinoma cribiforme & - & $1(0,47)$ \\
Carcinoma ductal & 1 & $1(0,47)$ \\
Carcinoma in situ & 1 & - & $1(0,47)$ \\
Comedocarcinoma & 1 & - & $1(0,47)$ \\
Fibrossarcoma & 1 & - & $1(0,47)$ \\
Sarcoma em tumor misto & 1 & - & $1(0,47)$ \\
Sarcoma & 1 & - & $1(0,47)$ \\
\hline Total & 199 & $213(100)$ \\
\hline Casos em que & & -
\end{tabular}

*Casos em que dois ou mais tipos histológicos estavam envolvidos

**Diagnóstico realizado por citologia

As neoplasias mamárias são em sua maioria hormônio dependentes em mamíferos, sendo o estrogênio um dos principais hormônios envolvidos na oncogênese desses tumores. A castração precoce previne o surgimento de neoplasias mamárias (Daleck et al., 2016; Fonseca \& Daleck, 2000). Em mulheres o fator hormonal também influencia na ocorrência de tumores mamários. Um estudo realizado na região sul revelou correlação positiva para o câncer de mama em mulheres que tiveram a menopausa tardia e fizeram uso de contraceptivos hormonais (Anjos et al., 2012). Assim como em mulheres, a chance de desenvolvimento de tumores mamários em animais submetidos a terapias de anticoncepção hormonal aumenta consideravelmente (Daleck et al., 2016; Fonseca \& Daleck, 2000). Outros fatores como alimentação, estilo de vida e predisposição genética possuem relação com a etiopatogenia do câncer de mama em humanos (Anjos et al., 2012).

O terceiro grupo mais frequente foi o de tumores dos órgãos sexuais e sistema reprodutor. Não houveram felinos diagnosticados com neoplasias deste grupo no período de estudo. Na tabela 6 observam-se os tumores do sistema reprodutor diagnosticados no HCV-UFPel em tal grupo.

O tumor mais frequente foi o tumor venéreo transmissível (64,5\%), seguido do seminoma (8\%) e sertolioma (6\%). O tumor venéreo transmissível é um tipo neoplásico capaz de ser transmitido através do transplante de células viáveis às mucosas, principalmente. Esse tipo de tumor ocorre principalmente em populações de cães errantes ou semi-domiciliados e não castrados. O principal sítio tumoral é a genitália, tanto do macho quanto da fêmea. Porém, podem ocorrer em outros locais como mucosa nasal, oral, olhos, pele e fazer metástases locais ou distantes. Apenas três tumores de mamíferos apresentam comportamento semelhante, sendo os outros dois o tumor facial do diabo da Tasmânia e um tumor semelhante de hamsters sírios (Ganguly et al., 2016; Peixoto et al., 2016). Uma parcela dos animais atendidos no HCV-UFPel são errantes, trazidos pela prefeitura da cidade de Pelotas ou então semidomiciliados. Tais fatos podem explicar a ocorrência relativamente alta de tal neoplasia. 
Tabela 6. Tumores do sistema reprodutor em cães diagnosticados no HCV-UFPel durante o período de 2013 a 2017

\begin{tabular}{lcc}
\hline Diagnóstico & Caninos, $\mathrm{n}$ & Frequência, \% \\
\hline Tumor venéreo transmissível vaginal & 32 & 42,10 \\
Tumor venéreo transmissível peniano & 17 & 22,37 \\
Sertolioma & 8 & 10,53 \\
Tumor de células intersticiais & 6 & 7,9 \\
Carcinoma prostático & 2 & 2,63 \\
Carcinoma ovariano & 2 & 2,63 \\
Seminoma & 2 & 2,63 \\
Carcinoma de células escamosas vulvar & 1 & 1,32 \\
Hiperplasia prostática & 1 & 1,32 \\
Leiomioma prostático & 1 & 1,32 \\
Leiomioma uterino & 1 & 1,32 \\
Leiomiossarcoma uterino & 1 & 1,32 \\
Leydignoma & 1 & 1,32 \\
Lipoma vaginal & 1 & 1,32 \\
\hline Total & 76 & 100 \\
\hline
\end{tabular}

O seminoma e o sertolioma são tipos neoplásicos testiculares bastante comuns. Tais tumores têm sua maior incidência em animais criptorquidas e com hérnias testiculares devido às alterações do microambiente testicular. Dentre os tumores testiculares, o sertolioma apresenta maiores chances de produzir metástases, entre 10 e 14\%. As metástases de seminoma podem ocorrer de 6 a $11 \%$ dos casos. Ambos os tumores são facilmente prevenidos com a castração (Daleck et al., 2016; Domingos \& Salomão, 2011).

Em seguida, o próximo grupo foi o de tumores do sangue e sistema hemolinfático, correspondendo a 6,54\% da casuística. A tabela 7 mostra os diagnósticos de tal grupo no período de estudo.

Tabela 7. Tumores do sangue e sistema hemolinfático de cães e gatos diagnosticados no HCV-UFPel durante o período de 2013 a 2017

\begin{tabular}{lccc}
\hline Diagnóstico & Caninos, $\mathrm{n}$ & Felinos, $\mathrm{n}$ & Total, $\mathrm{n}(\%)$ \\
\hline Linfoma & 17 & 12 & $29(58)$ \\
Hemangiossarcoma esplênico & 12 & - & $12(24)$ \\
Hiperplasia nodular esplênica & 4 & - & $4(8)$ \\
Hemangioma & 1 & - & $1(2)$ \\
Hemangiopericitoma cutâneo & 1 & - & $1(2)$ \\
Nódulo fibrohistiocítico esplênico & 1 & - & $1(2)$ \\
Sarcoma esplênico & 1 & 1 & $1(2)$ \\
Timoma & - & 13 & $50(100)$ \\
\hline Total & 37 & 2
\end{tabular}

O linfoma foi a neoplasia do sistema hemolinfático mais frequente corroborando com o descrito por Withrow et al. (2013). O linfoma é uma neoplasia caracterizada pela proliferação de linfócitos malignos e pode ocorrer em diversos tecidos pela contínua migração dessas células pelos diferentes tecidos do organismo (Daleck et al., 2016).

O próximo grupo, dos tumores dos ossos, articulações, cartilagens e músculos, foi responsável por $3,4 \%$ da casuística estudada. A tabela 8 mostra os diagnósticos do referido grupo durante o período de estudo.

O osteossarcoma foi o tumor mais frequente neste grupo, representando $65,38 \%$ do total de diagnósticos. O osteossarcoma é um dos tumores ósseos mais comuns tanto em homens quanto em animais e é localmente invasivo e potencialmente metastático principalmente para os pulmões (Arthur et al., 2016). 
Tabela 8. Tumores dos ossos, articulações, cartilagens e músculos diagnosticados em cães e gatos no HCV-UFPel durante o período de 2013 a 2017

\begin{tabular}{lccc}
\hline Diagnóstico & Caninos, $\mathrm{n}$ & Felinos, $\mathrm{n}$ & Total, $\mathrm{n}(\%)$ \\
\hline Osteossarcoma & 16 & 1 & $17(65,38)$ \\
Rabdomiossarcoma & 4 & 1 & $5(19,23)$ \\
Condrossarcoma & 2 & - & $2(7,69)$ \\
Sarcoma de células sinoviais & 1 & - & $1(3,85)$ \\
Sarcoma ósseo & 1 & - & $1(3,85)$ \\
\hline Total & 24 & 2 & $26(100)$ \\
\hline
\end{tabular}

A casuística de tumores do sistema respiratório e órgãos intratorácicos teve uma frequência relativamente baixa, correspondendo a $2,88 \%$ do total de neoformações deste estudo. Na tabela 9 estão descritos os diagnósticos de tumores deste grupo.

Tabela 9. Tumores do sistema respiratório e órgãos intratorácicos diagnosticados em cães e gatos no HCV-UFPel durante o período de 2013 a 2017

\begin{tabular}{lccc}
\hline Diagnóstico & Caninos, $\mathrm{n}$ & Felinos, $\mathrm{n}$ & Total, $\mathrm{n}(\%)$ \\
\hline Adenocarcinoma nasal & 2 & - & $2(9,1)$ \\
Carcinoma bronquioloalveolar & 1 & 1 & $2(9,1)$ \\
Metástase pulmonar de carcinoma anaplásico de mama & 2 & - & $2(9,1)$ \\
Osteossarcoma nasal & 2 & - & $2(9,1)$ \\
Tumor venéreo transmissível nasal & 2 & - & $2(9,1)$ \\
Carcinoma pulmonar & 1 & - & $1(4,54)$ \\
Condrossarcoma nasal & - & 1 & $1(4,54)$ \\
Hemangioma laríngeo & 1 & - & $1(4,54)$ \\
Linfoma intranasal & - & 1 & $1(4,54)$ \\
Mesotelioma torácico & 1 & - & $1(4,54)$ \\
Metástase pulmonar de carcinoma tubular de mama & 1 & - & $1(4,54)$ \\
Metástase pulmonar de carcinossarcoma & 1 & - & $1(4,54)$ \\
Metástase pulmonar de melanoma & 1 & - & $1(4,54)$ \\
Pólipo nasal & - & 1 & $1(4,54)$ \\
Sarcoma histiocítico pulmonar & 1 & - & $1(4,54)$ \\
Sarcoma intranasal & 1 & - & $1(4,54)$ \\
Tumor de células pequenas pulmonar & 1 & - & $22(100)$ \\
\hline Total & 18 & 4 & \\
\hline
\end{tabular}

Os tumores torácicos, principalmente os pulmonares, constituem os tumores mais frequentes no mundo em humanos segundo o INCA. No Brasil, tais neoplasmas estão na lista dos mais frequentes após tumores de pele não melanoma, tumores mamários e tumores prostáticos. O principal fator epidemiológico associado aos tumores pulmonares primários em humanos é o fumo. Segundo Daleck et al. (2016), as neoplasias pulmonares primárias em cães e gatos são raras, e ainda não existem trabalhos que comprovem o aumento da incidência desse tipo de neoplasia em animais expostos ao fumo passivo. Porém, em humanos, os fumantes passivos apresentam até 1,5 vezes maior chance de desenvolver neoplasias pulmonares. No presente estudo, notou-se que as doenças pulmonares mais frequentes foram às metástases.

Já o grupo dos tumores da cavidade oral e faringe foram responsáveis por 2,75\% da casuística total. Na tabela 10 há a descrição dos diagnósticos e frequências encontradas neste grupo.

O neoplasma mais frequente deste grupo foi o melanoma oral, correspondendo a $28,58 \%$ da casuística. Em um estudo realizado por Ferro et al. (2004), os tumores malignos orais mais frequentes foram os melanomas, seguido pelos carcinomas de células escamosas e os fibrossarcomas. Além disso, no mesmo estudo, o épulis foi o tumor benigno mais comum. Os achados anteriormente descritos corroboram com os achados deste trabalho. 
Tabela 10. Tumores da cavidade oral e faringe diagnosticados em cães e gatos no HCV-UFPel, durante o período de 2013 a 2017

\begin{tabular}{lccc}
\hline Diagnóstico & Caninos, $\mathrm{n}$ & Felinos, $\mathrm{n}$ & Total, $\mathrm{n}(\%)$ \\
\hline Melanoma oral & 6 & - & $6(28,58)$ \\
Carcinoma de células escamosas oral & 2 & 1 & $3(14,3)$ \\
Épulis fibromatoso & 3 & - & $3(14,3)$ \\
Fibrossarcoma oral & 3 & - & $3(14,3)$ \\
Ameloblastoma & 2 & - & $2(9,50)$ \\
Tumor odontogênico & 2 & - & $2(9,50)$ \\
Fibro odontoma ameloblástico & 1 & - & $1(4,76)$ \\
Osteossarcoma oral & 1 & - & $1(4,76)$ \\
\hline Total & 20 & 1 & $21(100)$ \\
\hline
\end{tabular}

Os tumores do trato gastrointestinal e glândulas anexas corresponderam a 2,61\% da casuística total, casuística relativamente baixa. A tabela 11 descreve a frequência e número de diagnósticos deste grupo.

Os sarcomas intestinais foram os mais frequentes, seguido pelos adenocarcinomas intestinais. Segundo Withrow et al. (2013) os tumores intestinais são raros em animais, sendo que em um estudo realizado no Reino Unido a incidência de tumores alimentares foi de 210 a cada 100.000 animais.

Tabela 11. Tumores do trato gastrointestinal e glândulas anexas diagnosticados em cães e gatos no HCV-UFPel, durante o período de 2013 a 2017

\begin{tabular}{lccc}
\hline Diagnóstico & Caninos & Felinos & Total \\
& $\mathrm{n}$ & $\mathrm{n}(\%)$ \\
\hline Sarcoma intestinal & 10 & - & $10(50)$ \\
Adenocarcinoma intestinal & 1 & 1 & $2(10)$ \\
Adenoma intestinal & 1 & - & $1(5)$ \\
Carcinoma hepático & 1 & - & $1(5)$ \\
Carcinoma hepatocelular & 1 & - & $1(5)$ \\
Carcinoma do pâncreas exócrino & 1 & - & $1(5)$ \\
Colangiocarcinoma & 1 & - & $1(5)$ \\
Hiperplasia nodular hepática & 1 & - & $1(5)$ \\
Leiomioma gástrico & 1 & - & $1(5)$ \\
Linfoma alimentar & - & 1 & $1(5)$ \\
Sarcoma gástrico & - & 1 & $1(5)$ \\
Sarcoma hepático & 1 & - & $1(5)$ \\
\hline Total & 17 & 3 & $20(100)$ \\
\hline
\end{tabular}

Os tumores do sistema urinário também foram um grupo pouco frequente, correspondendo a $0,92 \%$ da casuística. Na tabela 12 apresentam-se os tipos histológicos diagnosticados neste grupo.

Tabela 12. Tumores do sistema urinário diagnosticados em cães no HCV-UFPel, durante o período de 2013 a 2017

\begin{tabular}{lcc}
\hline Diagnóstico & Caninos, $\mathrm{n}$ & Frequência, \% \\
\hline Carcinoma de células transicionais & 5 & 71,42 \\
Carcinoma renal tipo células claras & 1 & 14,29 \\
Hemangiossarcoma renal & 1 & 14,29 \\
\hline Total & 7 & 100 \\
\hline
\end{tabular}

O tumor mais frequente neste grupo foi o carcinoma de células transicionais. As neoplasias da vesícula urinária acometem cerca de $2 \%$ dos animais, sendo que os carcinomas de células transicionais são o tipo neoplásico mais frequente (Withrow et al., 2013). Outro grupo tumoral que apresentou baixa frequência de diagnósticos foi o grupo dos tumores dos olhos e anexos. Na tabela 13 estão apresentados os diagnósticos histopatológicos do grupo, bem como a frequência e o número de animais atendidos de cada espécie. Os tumores oculares apresentam frequência baixa tanto em cães quanto em gatos, apesar 
de causarem consequências desastrosas ao animal mesmo quando benignos, pois levam à perda da visão e do globo ocular na maioria das vezes (Withrow et al., 2013).

Tabela 13. Tumores dos olhos e anexos diagnosticados em cães e gatos no HCV-UFPel, durante o período de 2013 a 2017.

\begin{tabular}{lccc}
\hline Diagnósticos & Caninos, $\mathrm{n}$ & Felinos, $\mathrm{n}$ & Total, $\mathrm{n}(\%)$ \\
\hline Adenoma de meibomian & 1 & - & $1(20)$ \\
Fibrossarcoma palpebral & - & 1 & $1(20)$ \\
Melanoma de íris & - & 1 & $1(20)$ \\
Melanoma palpebral & 1 & - & $1(20)$ \\
Papiloma corneal & 1 & - & $1(20)$ \\
\hline Total & 3 & 2 & $5(100)$ \\
\hline
\end{tabular}

Os tumores do sistema nervoso apresentaram um pequeno número de casos no período estudado $(0,52 \%)$. A tabela 14 mostra os tumores do sistema nervoso diagnosticados no período. O mais frequente foi o neuroblastoma olfatório. Apenas caninos foram diagnosticados com tumores do sistema nervoso. Esses tumores podem ter sido subdiagnosticados no HCV-UFPel pela ausência de métodos de diagnósticos por imagem apropriada para tal na cidade de Pelotas, como tomografia e a ressonância magnética. Outro fator importante é o fato de que o diagnóstico ante mortem é bastante complicado pela dificuldade de acesso aos tumores.

Tabela 14. Tumores do sistema nervoso diagnosticados em cães no HCV - UFPel, durante o período de 2013 a 2017

\begin{tabular}{lcc}
\hline Diagnóstico & Caninos, $\mathrm{n}$ & Frequência, $\%$ \\
\hline Neuroblastoma olfatório & 2 & 50 \\
Astrocitoma & 1 & 25 \\
Tumor de bainha neural maligno & 1 & 25 \\
\hline Total & 4 & 100 \\
\hline
\end{tabular}

O grupo dos tumores retroperitoneais e peritoneais teve casuística baixa, correspondendo a $0,40 \%$ da casuística total. Na tabela 15 observam-se os tumores diagnosticados neste grupo, bem como o número de caninos e felinos acometidos e a frequência da ocorrência dos mesmos.

Tabela 15. Tumores retroperitoneais e peritoneais diagnosticados em cães e gatos no HCV-UFPel, durante o período de 2013 a 2017

\begin{tabular}{lcc}
\hline Diagnóstico & Caninos, $\mathrm{n}$ & Frequência, $\%$ \\
\hline Hemangiossarcoma retroperitoneal & 1 & 33,33 \\
Mesotelioma & 1 & 33,33 \\
Sarcoma indiferenciado & 1 & 33,33 \\
\hline Total & 3 & 100 \\
\hline
\end{tabular}

Os tumores das glândulas endócrinas corresponderam a 0,40\% da casuística estudada. Foi o grupo de tumores com menor frequência e apenas um tipo histológico foi encontrado, o carcinoma de tireoide. Apenas três caninos foram acometidos. Segundo Withrow et al. (2013), em um estudo realizado com 545 amostras de caninos com câncer de tireoide, $90 \%$ deles eram carcinomas ou adenocarcinomas.

\section{Conclusão}

Os grupos de neoplasmas cutâneos e mamários foram o mais frequente no presente estudo, assim como as atuais estimativas em humanos. Dessa maneira, pode-se concluir que há muitas semelhanças entre a oncologia veterinária e humana, permitindo que futuramente cães e gatos possam ser modelos de estudo da ocorrência natural do câncer beneficiando todas as espécies envolvidas.

\section{Referências bibliográficas}

Anjos, J. C. d., Alayala, A. \& Höfelmann, D. A. (2012). Fatores associados ao câncer de mama em mulheres de uma cidade do Sul do Brasil: estudo caso-controle. Cadernos Saúde Coletiva, 20(3):341-350. 
Arthur, E. G., Arthur, G. L., Keeler, M. R. \& Bryan, J. N. (2016). Risk of osteosarcoma in dogs after open fracture fixation. Veterinary Surgery, 45(1):30-35.

Benjamin, S. A., Lee, A. C. \& Saunders, W. J. (1999). Classification and behavior of canine mammary epithelial neoplasms based on life-span observations in beagles. Veterinary Pathology, 36(5):423-436.

Borsato, F. G. \& Almeida, E. d. F. P. (2009). Neoplasia de pele não melanoma: um agravo relacionado ao trabalho. Ciência, Cuidado e Saúde, 8(4):600-606.

Campisi, J. (2003). Cancer and ageing: rival demons? Nature Reviews Cancer, 3(5):339-349.

Daleck, C. R., Fonseca, C. S. \& Canola, J. C. (2016). Oncologia em cães e gatos. Rio de Janeiro: Roca.

De Nardi, A. B., Rodaski, S., Sousa, R. S., Costa, T. A., Macedo, T. R., Rodigheri, S. M., . . Piekarz, C. H. (2002). Prevalência de neoplasias e modalidades de tratamentos em cães, atendidos no hospital veterinário da Universidade Federal do Paraná. Archives of Veterinary Science, 7(2):15-26.

Demetriou, J. \& Foale, R. (2011). Oncologia em pequenos animais. EUA: Elsevier, 2011. EUA: Elsevier.

Domingos, T. C. S. \& Salomão, M. C. (2011). Meios de diagnóstico das principais afecções testiculares em cães: revisão de literatura. Revista Brasileira de Reprodução Animal, 35(4):393-399.

Ferreira Neto, J. M., Viana, J. M. M. E. S. \& Magalhães, L. M. (1977). Patologia clínica veterinária: Rabelo e Brasil.

Ferro, D. G., Lopes, F. M., Venturini, M. A. F. A., Correa, H. L. \& Gioso, M. A. (2004). Prevalência de neoplasias da cavidade oral de cães atendidos no Centro Odontológico Veterinário-Odontovet ${ }^{\circledR}-\mathrm{SP}-$ entre 1994 e 2003. Arquivos de Ciências Veterinárias e Zoologia da UNIPAR, 7(2):129-133.

Fonseca, C. S. \& Daleck, C. R. (2000). Neoplasias mamárias em cadelas: influência hormonal e efeitos da ovário-histerectomia como terapia adjuvante. Ciência Rural, 30(4):731-735.

Ganguly, B., Das, U. \& Das, A. K. (2016). Canine transmissible venereal tumour: a review. Veterinary and Comparative Oncology, 14(1):1-12.

Gjerstorff, M. L. (2011). The Danish cancer registry. Scandinavian Journal of Public Health, 39(7):42-45.

Grüntzig, K., Graf, R., Hässig, M., Welle, M., Meier, D., Lott, G., . . Boo, G. (2015). The Swiss canine cancer registry: a retrospective study on the occurrence of tumours in dogs in Switzerland from 1955 to 2008. Journal of Comparative Pathology, 152(2-3):161-171.

Hayes Junior, H. M. (1978). The comparative epidemiology of selected neoplasms between dogs, cats and humans. A review. European Journal of Cancer, 14(12):1299-1308.

Instituto brasileiro de geografia e estatística. População de Animais de Estimação no Brasil. Disponível em: $\quad$ http://www.agricultura.gov.br/assuntos/camaras-setoriais-tematicas/documentos/camarastematicas/insumos-agropecuarios/anos-anteriores/ibge-populacao-de-animais-de-estimacao-nobrasil-2013-abinpet-79.pdf/view. Acesso em 25 de julho de 2018.

Kavuluru, R., Hands, I., Durbin, E. B. \& Witt, L. (2013). Automatic extraction of ICD-O-3 primary sites from cancer pathology reports. AMIA Summits on Translational Science Proceedings, 2013112-116.

Lima, S. R., Stocco, M. B., Rondelli, L. A. S., Silva, G. S., Lopes, R. S., Furlan, F. H., . . Pescador, C. A. (2018). Neoplasmas cutâneos em cães: 656 casos (2007-2014) em Cuiabá, MT. Pesquisa Veterinária Brasileira, 38(7):1405-1411.

Merlo, D. F., Rossi, L., Pellegrino, C., Ceppi, M., Cardellino, U., Capurro, C., . . Tanara, G. (2008). Cancer incidence in pet dogs: findings of the Animal Tumor Registry of Genoa, Italy. Journal of Veterinary Internal Medicine, 22(4):976-984.

Núñez, O. L. \& Bouda, J. (2007). Patología Clínica Veterinaria (Vol. 1. ed). Mexico: FMVZ-UNAM.

Peixoto, P. V., Teixeira, R. S., Mascarenhas, M. B., França, T. N., Azevedo, S. C. S., Reinacher, M., . . . Ramadinha, R. R. (2016). Formas atípicas e aspectos clínico-epidemiológicos do tumor venéreo transmissível canino no Brasil. Brazilian Journal of Veterinary Medicine, 38(2):101-107.

Rowell, J. L., McCarthy, D. O. \& Alvarez, C. E. (2011). Dog models of naturally occurring câncer. Trends in Molecular Medicine, 17(7):380-388.

Souza, T. M., Fighera, R. A., Irigoyen, L. F. \& Barros, C. S. L. (2006). Estudo retrospectivo de 761 tumores cutâneos em cães. Ciência Rural, 36(2):555-560.

Withrow, S. J., Page, R. \& Vail, D. M. (2013). Small Animal Clinical Oncology-E-Book. St. Louis Missouri: Elsevier Health Sciences.

Recebido: 6 de março, 2019.

Aprovado: 2 de abril, 2019.

Publicado: 29 de abril, 2019

Licenciamento: Este artigo é publicado na modalidade Acesso Aberto sob a licença Creative Commons Atribuição 4.0 (CC-BY 4.0), a qual permite uso irrestrito, distribuição, reprodução em qualquer meio, desde que o autor e a fonte sejam devidamente creditados. 